\title{
Possible Transient Benefits of Epstein Barr Virus Infection in Three Subjects with Established Type 1 Diabetes
}

Willem M Kühtreiber, Sophie L Leung, Limei Wang, Elise Hsu, Peter E Reinhold III, Menghan Zhao, Hui Zhang, Douglas E Burger and Denise L Faustman*

Immunobiology Laboratories, Massachusetts General Hospital, Harvard Medical School, USA

\begin{abstract}
Tumor necrosis factor (TNF) is a novel immunotherapy for type I diabetes because it selectively kills insulin autoreactive T-cells, which enables recovery of insulin production by pancreatic islet cells. The TNF inducer Bacillus Calmette-Guerin (BCG) also has therapeutic value by activating innate immunity that beneficially modifies the course of type 1 diabetes (T1D) in the same manner as TNF. Epstein Barr Virus (EBV) infection is also an inducer of TNF. This observational study was undertaken to determine whether EBV has the same beneficial effects as BCG in a recent clinical trial. We describe three cases of long-term diabetic subjects with active mononucleosis that were followed for 15 weeks to determine the impact of EBV infection on established T1D. In comparison to non-EBVinfected long-term diabetics, EBV transiently diminished the autoimmune response in two of three cases. EBV infection triggered rapid increase of circulating insulin-B autoreactive T-cells whose striking loss of CD8 marker indicated that the cells were injured or apoptotic. EBV infection also caused a transient surge in the secretion of C-peptide, a marker for pancreatic insulin secretion. EBV acts like BCG in eliciting innate immunity and beneficially modifying the course of T1D.
\end{abstract}

Keywords: Immunotherapy; Infection; Immune adjuvants; Type 1 diabetes

Abbreviations: TNF: Tumor Necrosis Factor; BCG: Bacillus Calmette-Guerin; TNF: Tumor Necrisis Factor; T1D: Type 1 Diabetes; EBV: Epstein Barr Virus

\section{Introduction}

Research over the last fifteen years in the non-obese diabetic (NOD) mouse and in humans with type 1 diabetes has uncovered a therapeutic role of the cytokine tumor necrosis factor (TNF). TNF, whether directly administered or indirectly boosted with immune adjuvants such as Bacillus Calmette-Guérin [1] or infectious diseases $[2,3]$, acts as a novel immunotherapy. The systemic increase of TNF results in pancreas regeneration with the recovery of C-peptide [4-6]. C-peptide is the protein co-secreted with insulin and a sensitive method to measure insulin secretion from the pancreas in the presence of exogenously administered insulin. The molecular mechanisms behind TNF action are multi-factorial. First, TNF directly kills autoreactive T-cells [7]. Second, TNF induces beneficial T-regulatory (Treg) cells, the cells that dampen the function of autoreactive cytotoxic T-cells [8]. Lastly TNF augments spontaneous islet regeneration of the pancreas $[5,9]$. In vitro studies of human diabetic T-cells show that brief TNF exposures selectively kill autoreactive T-cells and induce proliferation of beneficial CD4 Treg cells [7].

In mammals, high-dose administration of TNF is systemically toxic, especially in disease settings where high systemic TNF may already exist [10]. An alternative approach is to use safe infectious agents that elicit the host to release TNF. This host immune response to infection is often referred to as innate immunity. Indeed, the Mycobacterium bovis Bacillus Calmette-Guerin (BCG) vaccine induces release of host TNF. This avirulent strain of tuberculosis led to the discovery of the host cachexia response due to cachectin, which was shown to be identical to the cytokine TNF once molecular cloning was complete.

During the conduct of a double-blinded placebo-controlled trial administering two doses of BCG for treatment of type 1 diabetes (T1D), it was discovered that one placebo control had immune responses that mimicked the BCG diabetic responses. The control subject had an acute Epstein Barr Virus (EBV) injection. The EBV infection response, albeit in one subject, provided evidence that infectious agents other than Mycobacterium can activate innate immunity in long-term diabetic subjects and beneficially modify the disease course. EBV induces host protective TNF secretion as a potent anti-viral cytokine [11], just like BCG and a variety of other bacterial and viral infections $[12,13]$. Similar to the BCG treated subjects, the EBV infected subject showed the occurrence of dead autoreactive T-cells, induction of Treg cells, and a transient increase in pancreatic insulin secretion, monitored by a sensitive C-peptide assay [6].

Previous data show that treatment of NOD mice or diabetic humans with BCG (or the non-cGMP equivalent Complete Freund's Adjuvant (CFA)) results in a transient increase in dead autoreactive T-cells. In the NOD mouse, pancreas-residing autoreactive T-cells are observed to undergo apoptosis directly on top of the insulin-secreting islets in the pancreas with TNF, BCG or CFA [14]. In humans, the impact of TNF on autoreactive T-cells can be monitored by the rapid release of dead autoreactive T-cells into the circulation [6]. Increased TNF or TNFR2 agonistic antibodies are known to cause apoptosis of murine and human diabetic autoreactive T-cells in culture [7].

*Corresponding author: Denise L. Faustman, Immunobiology Laboratories Massachusetts General Hospital, Harvard Medical School, Building 149, 13th Street, Room 3602, Boston, MA 02129, USA, E-mail: faustman@helix.mgh.harvard.edu

Received September 28, 2013; Accepted November 13, 2013; Published November 18, 2013

Citation: Kühtreiber WM, Leung SL, Wang L, Hsu E, Reinhold PE III, et al. (2013) Possible Transient Benefits of Epstein Barr Virus Infection in Three Subjects with Established Type 1 Diabetes. J Diabetes Metab 4: 309. doi:10.4172/21556156.1000309

Copyright: ( 2013 Kühtreiber WM, et al. This is an open-access article distributed under the terms of the Creative Commons Attribution License, which permits unrestricted use, distribution, and reproduction in any medium, provided the original author and source are credited. 
To understand and characterize further the impact of EBV infection on diabetic disease course, we now present three type 1 diabetic subjects followed in the clinic at frequent intervals during their new-onset EBV infection. We show the impact of an acute EBV infection on established type 1 diabetes and on the release of dead or injured $\mathrm{T}$ cells.

\section{Materials and Methods}

\section{Subjects}

Patients with T1D were recruited at the Massachusetts General Hospital. We routinely screen diabetic patients to characterize the course of disease and to exclude subjects with potentially interfering medical conditions. During screening we identified three patients with long-term T1D who presented with recent onset of mononucleosis as diagnosed by their primary care physicians and confirmed by the presence of serum EBV antibodies. Diabetics without acute EBV infection $(\mathrm{N}=66)$ were used in this study as a reference population. All patient and control blood was drawn into BD Vacutainer ${ }^{\mathrm{TM}}$ tubes (BD, Franklin Lakes, NJ) containing EDTA.

\section{Ethics statement}

The study was approved by the Massachusetts General Hospital Institutional Review Board (IRB Protocol No. 2001P-001379). Written consent was obtained from all blood donors.

\section{Detection of epitope-specific CD8 T-cells}

For the detection of specific subpopulations of T-cells with restricted antigen specificity, commercially available HLA Class I reagents loaded with small peptide fragments were utilized. These commercial reagents are commonly referred to as Tetramers (MBL International, DesPlaines IL., formerly Beckman Coulter, Fullerton, CA) or Dextramers (Immudex, Fairfax, VA). The two T-cell detection methods differ in the backbone structures of the detecting reagent but not in the binding specificity to autoreactive T-cells. Tetramer or dextramer reagents are purchased fluorescently labeled for detection of the bound reagent to antigen specific T-cell with a flow cytometer.

For the studies contained in this paper, two types of antigenspecific T-cells were detected using tetramers and dextramers loaded with peptide sequences from epitopes of EBV or of pancreatic beta cells that are known to be recognized by CD8 T cells, i.e., EBV-specific (HLA class I loaded peptide; GLCTLVAML) or insulin-B specific (HLA class I loaded Insulin-B chain; HLVEALYLV). For background fluorescence of T-cells a matched HLA class I structure was loaded with an irrelevant peptide. The peptide sequence of this negative control is kept proprietary by the companies, but does not occur in nature (Beckman Coulter, Immudex).

\section{Isolated CD8 T-cell method}

For Subject \#1 we isolated CD8 T cells from fresh blood for flow cytometry. We used the "Detach-a-Bead" CD8 positive isolation kit from Life Technologies (Carlsbad, CA). This kit uses paramagnetic beads coated with anti-CD8 antibody [15]. The beads were allowed to attach for $1 \mathrm{hr}$ at room temperature under continuous agitation on a tumbler. The bead/cell complexes were then immobilized using a magnet and any unbound (non-CD8) cells removed by repeated washing with HBSS (Hank's Balanced Salt Solution without calcium and magnesium, Invitrogen, Grand Island, NY) containing 2\% FBS (fetal bovine serum). The beads were then detached from the remaining CD8 cells using a Detach-A-Bead reagent supplied in the isolation kit. This reagent is a polyclonal antibody directed against the antigen recognition site of the CD8 antibody coated on the beads. It detaches the antibody/bead complex from the cells by means of competition for the CD8 antibody binding site, essentially leaving a virgin cell.

Isolated CD8 T-cells were then labeled with PE (phycoerythrin) labeled tetramers or dextramers (20 min, room temperature(RT), in the dark) and subsequently with APC-anti-CD8 antibodies (10 minutes at RT in the dark; clone SK1, BD Biosciences, San Jose, CA) to determine purity of the isolated cell preps. The samples were then fixed with HBSS $0.1 \%$ formaldehyde buffer, washed with HBSS, and re-suspended in HBSS/ $0.05 \%$ formaldehyde for flow cytometry.

\section{Whole blood method}

The whole blood method was used for Subject \#2 and \#3. Blood samples were first washed with 50 volumes of HBSS containing $2 \%$ FBS. They were then labeled with the PE-tetramers or PE-dextramers (20 min at RT in the dark) and subsequently with APC-anti-CD8 antibodies (10 minutes at RT in the dark) to enable gating on CD8 T-cells. The samples were then simultaneously lysed and fixed with $\mathrm{NH}_{4} \mathrm{Cl}$ /formaldehyde buffer, washed with HBSS, and re-suspended in HBSS/formaldehyde for flow cytometry.

\section{Flow cytometry}

Cells were analyzed using a FACSCalibur flow cytometer (BD Biosciences, San Jose, CA) and data collected in list mode. Data analysis was performed using Cell Quest software (BD Biosciences). Flow gates were set "open" for inclusion of all cells. The open gate included cells of all sizes, but excluded cell debris, red blood cells, fragmented cells, and apoptotic bodies. The percentage CD8 T-cells was defined as the ratio of the number of CD8 positive events and the total number of events in the lymphocyte gate.

\section{ELISA}

C-peptide was determined by Elisa in blood serum. Serum was prepared by centrifugation after clotting and stored at $-80^{\circ} \mathrm{C}$. The ultrasensitive C-peptide Elisa was from Mercodia (Uppsala, Sweden). The kit was used according to the manufacturers' instructions. Serum levels of VCA IgM, Early Antigen D, and EBNA were determined by Massachusetts General Hospital Clinical Laboratory Services.

\section{Statistics}

Statistical significance was determined using the unpaired, onetailed Student's t-test at a confidence level of 0.05 . We used the one sided Kolmogorov-Smirnov sample test to compare the distribution of each patient with the reference samples (Figures 1-3).

\section{Results}

\section{Clinical profiles}

In the course of routine visits of T1D patients, we identified three patients who presented with recent onset EBV infection, also known clinically as mononucleosis or "mono". We followed these patients closely for at least 15 weeks after presentation. Recent onset EBV infection offers an opportunity to study the prevalence and traits of autoimmune and EBV-specific T-cells both during and after clinical symptoms of EBV infection. It also offers the opportunity to document the reproducibility of the past observation in a clinical trial of BCG treatment that EBV infection caused a transient increase in insulin production by the pancreas, measured as co-secreted C-peptide [6]. 
The EBV infections were first clinically diagnosed by standard serologic methods and symptoms. The clinical characteristics of these subjects and of the reference population are summarized in Figure 1A. All subjects had established Type 1 diabetes, with durations of 6,19 , and 30 years and elevated HbA1c. Two of the subjects (Subjects 1 and 2) were positive for glutamic acid decarboxylase (GAD65) autoantibody, which is an islet-specific marker for T1D.

\section{Time course of EBV infection}

After presenting with symptoms and a clinical diagnosis of EBV infection, we performed more detailed EBV antibody serology to determine the details of the time course of the infection. We wanted to estimate the time of onset of the infection relative to the sequential blood studies performed here (Figure 1B).

Isotypes of EBV antibodies to different parts of the EBV viral particle peak at different times after infection [16]. We used a combination of VCA IgM, Early Antigen D, and EBNA. These markers peak at 0-6 weeks, 4-8 weeks, and 6-8 weeks after EBV infection, respectively (Figure 1B). All subsequent data reported in this study, especially timelines of immunologic events, were plotted and extrapolated to an infection date of " 0 ". For Subject 1, we estimated that the presentation time for the present study was week 2 after infection, for Subject 2 the presentation time was week 4 after infection, and for Subject 3 the presentation time was week 2 after infection.

\section{Detection of EBV-specific CD8 T-cells}

Although the typical clinical method of diagnosing EBV infections involves antibody testing, it is possible in a research setting to confirm infection by direct monitoring of newly created EBV-specific T-cells. We used EBV-specific tetramers, i.e., HLA class I proteins loaded with a synthetic peptide sequence GLCTLVAML. This allowed us to detect EBV-specific CD8 T-cells using flow cytometry (Figures 1C and 1D). Although a slightly different method of detection of these EBV-specific T-cells was utilized in Subject 1, versus Subjects 2 and 3 (isolated CD8 T-cells versus direct observations of CD8 T-cells in lysed blood), all subjects had an early rise in EBV-specific T-cells above background levels of fluorescence (Figure 1D).

\section{Time course of $\mathrm{C}$-peptide in EBV-infected subjects}

To determine the impact of EBV infections on insulin secretory capacity, we monitored serial serum C-peptide levels for at least 15 weeks after the subjects with EBV presented to the research clinic. A simultaneously studied reference population of non-EBV infected longterm diabetics was similarly monitored for 15 weeks. The monitoring of the reference subjects for fasting morning C-peptide demonstrated the subject and assay variability.

C-peptide in EBV-infected Subjects 1 and 2 showed a statistically significant increase of $\mathrm{p}=0.04$ and $\mathrm{p}=0.0013$ over that in the reference population, respectively. Subject 3 showed no significant increase in pancreatic C-peptide (Figure 2).

\section{Impact of EBV on Insulin-B Autoreactive T-cells in Type 1 diabetics}

The detection of peripherally released injured or dead autoreactive $\mathrm{T}$ cells after BCG or EBV is an indicator of disease modification. To determine whether EBV infection similarly kills or injures autoreactive T-cells, we quantified insulin-B autoreactive CD8 T-cells in the peripheral blood of the three recently EBV-infected subjects compared to uninfected long-term diabetic subjects. Tracking of insulin-B autoreactive T-cells in long-term diabetics is feasible. With sensitive monitoring methods about $41 \%$ (21 out of 51 ) of randomly recruited long-term type 1 subjects had detectable insulin-B autoreactive T-cells $(0.28 \%$ to $0.65 \%$, Figure $3 \mathrm{~A})$. The same type 1 diabetic CD8 T-cells stained with negative dextramer reagents exhibited a background signal range of $0.19 \%$ to $0.27 \%$ (Figure $3 \mathrm{~A}$ ).

As previously shown for long-term diabetics exposed to TNF inducing infections, the EBV infected subjects exhibited an overabundance of insulin-B autoreactive T-cells in peripheral blood after infection. The mean percentage of insulin-B autoreactive T-cells in EBV-infected subjects was greater than that in the negative background staining $(\mathrm{p}=0.002)$, as well as in a matched population of uninfected reference diabetics $(\mathrm{p}=0.02)$. This finding supports the conclusion that

A.

\begin{tabular}{lccccc}
\multicolumn{6}{l}{ Table 1. Clinical characteristics of subjects and reference population } \\
\hline Characteristic & Subject 1 & Subject 2 & Subject 3 & $\begin{array}{c}\text { Reference Subjects } \\
\text { (Average) }\end{array}$ & (Range) \\
\hline Sex & $\mathrm{M}$ & $\mathrm{F}$ & $\mathrm{F}$ & $29 \% \mathrm{~F}$ & \\
Age $(\mathrm{y})$ & 40 & 17 & 21 & 49 & $29-71$ \\
Age of T1D onset $(\mathrm{y})$ & 17 & 11 & 2 & 31 & $1-52$ \\
T1D duration $(\mathrm{y})$ & 23 & 6 & 19 & 18 & 3.43 \\
HDA1C $(\%$ ) & 6.2 & 8.0 & 9.6 & 7.0 & $6.2-9.2$ \\
GAD65 status & positive & positive & negative & $\mathrm{n} / \mathrm{a}$ & $\mathrm{n} / \mathrm{a}$ \\
\hline
\end{tabular}

B.

\begin{tabular}{|c|c|c|c|c|}
\hline Antibody & VCA IgM & Early Antigen D & EBNA & \\
\hline $\begin{array}{l}\text { Time after } \\
\text { EBV }\end{array}$ & $0-6$ Weeks & 4-8 Weeks & $\begin{array}{l}6-8 \text { Weeks } \\
\text { Sustained }\end{array}$ & $\begin{array}{l}\text { Estimated } \\
\text { Duration of EBV } \\
\text { Infection }\end{array}$ \\
\hline $\begin{array}{l}\text { Subject } 1 \\
\text { Subject } 2\end{array}$ & $\begin{array}{l}\text { Negative } \\
\text { Positive }\end{array}$ & $\begin{array}{l}\text { Positive } \\
\text { Positive }\end{array}$ & $\begin{array}{c}\text { n/a } \\
\text { Negative }\end{array}$ & $\begin{array}{l}2 \text { weeks } \\
4 \text { weeks }\end{array}$ \\
\hline Subject 3 & Positive & Negative & Negative & \\
\hline
\end{tabular}

c.
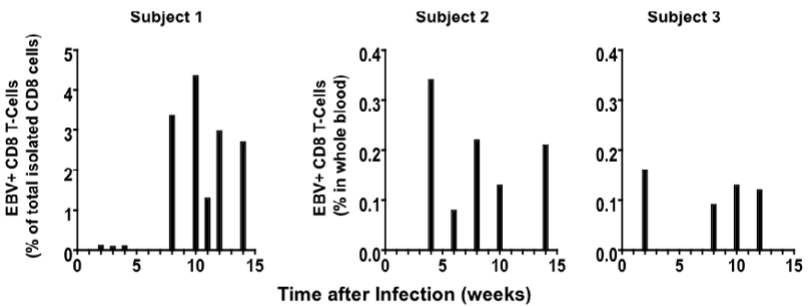

D.
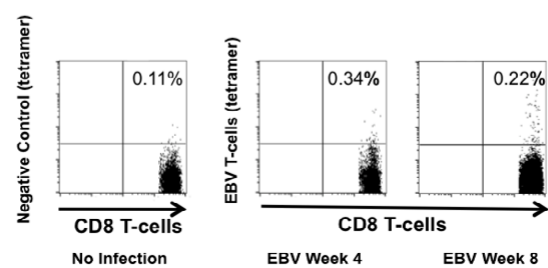

Figure 1: Clinical characteristics and time course of EBV infection. A Summary of clinical characteristics for the three EBV subjects. B. Results for EBV antibody testing on blood samples drawn during the first visit for the three EBV subjects and correlation with estimated start of EBV infection. C. Time course of the appearance of EBV-specific T-cells in Subject 1, Subject 2 and Subject 3 . As detailed in the Materials and Methods, the staining for EBV reactive T-cells in Subject 1 was performed on isolated CD8 T-cells; the staining for EBV reactive T-cells for Subject 2 and Subject 3 was performed on whole blood lysates with CD8 T cells identified with CD8 antibody. In the graphs, time point 0 indicates the estimated occurrence of EBV infection based on the timing of the appearance of antibodies directed against EBV. D. Representative dot plots of flow cytometry results. Level of non-specific tetramer binding by CD8 T-cells was determined using a tetramer loaded with an irrelevant peptide sequence (Negative Control, left panel). The middle and right panels show examples of positive staining using EBV-specific tetramers. 
infections that boost TNF result in the release of these autoreactive T-cells into the circulation.

\section{Newly released insulin-B autoreactive T-cells have abnormally low CD8 density}

A further analysis of the insulin-B autoreactive T-cells in long-term diabetics infected with EBV virus revealed some additional distinctive features of the antigen-specific CD8 T-cells (Figure 3B). Not only were there more insulin-B autoreactive CD8 T-cells in the circulation after EBV infection, but also their density of the CD8 marker was dramatically lower. As shown in Figure 3B, in three characteristic histograms, the CD8 mean log fluorescent density was 1408, 1157 and 1516 for the insulin-B autoreactive T-cells in EBV-infected subjects. In contrast, for insulin-B autoreactive T-cells from uninfected longterm diabetic subjects, the mean log antigen density of the CD8 marker was 2976, 4003, and 4948. The lower density of CD8 proteins in the EBV-infected subjects was not a generalized trend for all antigenspecific T-cells but specific for only the autoreactive T-cells. For these same infected subjects the log CD8 T-cell density was normal for the EBV-specific T-cells. The CD8 protein density on the cell surface was 4308,3530 and 4416 , values that are similar to the density of the CD8 proteins on insulin-B autoreactive T-cells of uninfected diabetics (Figure 3B). Loss of CD8 marker is indicative of cellular damage or apoptosis of T-cells [17].

\section{Discussion}

In this paper we describe three case studies of Type 1 diabetics that presented with acute EBV infections. Overall this study reinforces our past observation that infection with EBV transiently diminishes the autoimmune response in type 1 diabetes, which facilitates the brief recovery of pancreatic function. EBV infection causes rapid release into the circulation of increased numbers of preformed circulating insulin-B autoreactive T-cells. These autoreactive T-cells express dramatically lowered levels of the CD8 protein, indicative of injury or apoptosis. The EBV infection also causes transient and modest increases in insulin secretion as measured by C-peptide from the pancreas.

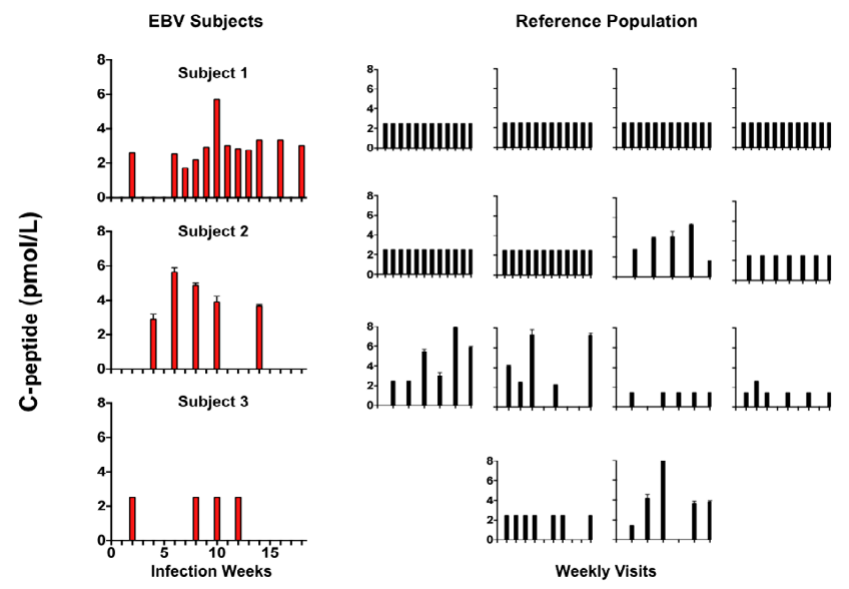

Figure 2: Time course of C-peptide levels in the EBV-infected subjects versus a reference population of Type 1 Diabetics without active EBV infection. Subjects 1 and 2 showed a transient increase in C-peptide that was statistically significant as compared to the reference population $(p<0.04$ and $p=0.00134$ respectively). Subject 3 did not show increased $C$-peptide levels.

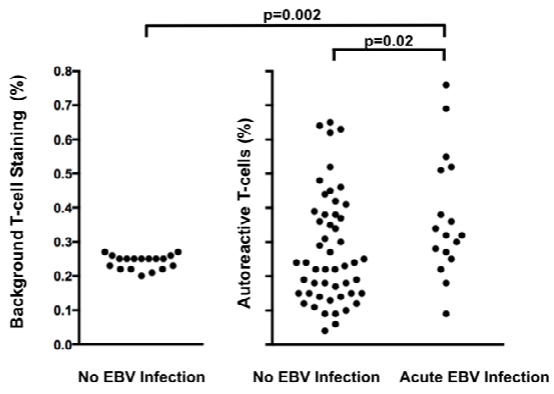

B.

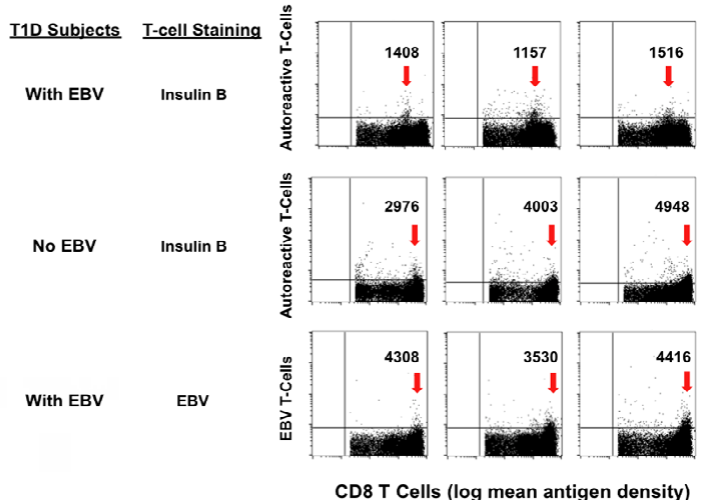

Figure 3: EBV infection results in increased presence of autoreactive T-cells with reduced CD8 antigen density. A. Increased presence of circulating insulin-B autoreactive T-cells after EBV infection in T1D as compared to the levels of autoreactive T-cells in an uninfected T1D subjects. Background fluorescence for peripheral T-cells as shown is $0.19 \%$ to $0.27 \%$.(left panel) B. Reductions in CD8 antigen density were specific for the newly appearing insulin-B autoreactive T-cells of the EBV-infected subjects. Long-term type 1 diabetics without EBV infection had smaller numbers of peripheral insulin-B autoreactive T-cells, but the density of the CD8 protein was in the norma range. Also the EBV-specific T-cells from the EBV-infected subjects also had a normal density of CD8 protein.

Subjects 1 and 2 had a more dramatic response to the EBV infection than Subject 3. Subject 3 did not have a statistically significant increase in C-peptide production and had only a modest degree of EBVspecific T-cell generation. We can speculate why Subject 3 appears to be different from the other long-term diabetics who displayed more beneficial immune and pancreatic responses to EBV. Subject 3 was GAD65 negative at baseline, a sign of no remaining pancreas activity. Subject 3 had an age of onset of diabetes at 2 years of age, a trait known to cause more rapid decline in pancreas activity, thus explaining why autoantibody to the insulin secreting islet tissue, GAD65, was negative. Lastly and perhaps most significantly, Subject 3 had a starting $\mathrm{HbAlc}$ that was high, 9.6. We know from past NOD mouse studies that the pancreas does not regenerate when control of blood sugar is poor during the treatment period with TNF induction [9]. Therefore we speculate the poor blood sugar control during the infection in Subject 3 could have hampered the regenerative process. A pancreas with no remaining insulin-secreting islets for a GAD65 autoantibody response may characterize patients who might not benefit from TNF induction. This inference may be important for the design of future clinical trials in long-term diabetics.

A new T-cell observation was discovered in this observational study. As shown in Figure 3B, the newly released autoreactive T-cells 
after EBV infection have a dramatically decreased density of CD8 proteins on their surface. The decrease in CD8 proteins was neither a trait of the newly appearing EBV-specific T-cells nor a trait of the general CD8 T-cell populations in the circulation. Also the circulating insulin-B autoreactive T-cells in long-term diabetic subjects without an EBV infection had normal CD8 protein density. What does this mean? Past studies have shown that TNF induction in long-term diabetics rapidly releases damaged and dying autoreactive T-cells into the circulation [6]. It is known that as CD8 T-cells start to die the density of the surface markers starts to fall since re-synthesis of CD8 is not possible [17]. Therefore we suggest that the dramatic drop in CD8 protein density is a trait of those T-cells damaged or dying from the EBV infection-induced TNF release. Since the drop in density of the CD8 marker is large, this may be an easier method to measure death of autoreactive T-cells than more complicated T-cell viability assays used in the past $[6,9,15]$. As observed by past in vitro studies of antigen specific T-cells in type 1 diabetics, TNF has target specificity for killing only the autoreactive $\mathrm{T}$-cells, not other populations of antigen-activated T-cells, here demonstrated by the hardiness of antigen specific EBV T-cells from type 1 diabetics to resist cell death [7].

While it is limited to only three cases, this observational study of the impact of EBV infections on diabetic autoimmunity lends support to the "hygiene hypothesis". This hypothesis holds that reduction in early-life infections is associated with the worldwide rise in immune disorders, including T1D [18]. The hygiene hypothesis attributes the growing incidence of autoimmunity to fewer infections, better sanitation, greater antibiotic use, and improved socio-economic indices [19-23]. With fewer infections there are likely fewer host inductions of TNF. TNF is known to protect the host through autoreactive $\mathrm{T}$ cell death [7] and also induction of Tregs [24-26]. Our observational study and previous studies $[6,27]$ extend the epidemiologically-driven "hygiene hypothesis" by demonstrating that EBV infection or deliberate reintroduction of attenuated tuberculosis bacteria results in amelioration of autoimmunity even years after disease onset.

\section{Acknowledgements}

We are grateful for the editorial assistance of Dr. Miriam Davis as well as for manuscript and reference preparations by Ms. Lynne Murphy.

\section{References}

1. Oliveira MM, Charlab R, Pessolani MC (2001) Mycobacterium bovis BCG but not Mycobacterium leprae induces TNF-alpha secretion in human monocytic THP-1 cells. Mem Inst Oswaldo Cruz 96: 973-978.

2. Gosselin J, Flamand L, D'Addario M, Hiscott J, Menezes J (1992) Infection of peripheral blood mononuclear cells by herpes simplex and Epstein-Barr viruses. Differential induction of interleukin 6 and tumor necrosis factor-alpha. $\mathrm{J}$ Clin Invest 89: 1849-1856.

3. Lay JD, Tsao CJ, Chen JY, Kadin ME, Su IJ (1997) Upregulation of tumor necrosis factor-alpha gene by Epstein-Barr virus and activation of macrophages in Epstein-Barr virus-infected T cells in the pathogenesis of hemophagocytic syndrome. J Clin Invest 100: 1969-1979.

4. Faustman D (1993) Mechanisms of autoimmunity in type I diabetes. J Clin Immunol 13: 1-7.

5. Kodama S, Kühtreiber W, Fujimura S, Dale EA, Faustman DL (2003) Islet regeneration during the reversal of autoimmune diabetes in NOD mice. Science 302: 1223-1227.
6. Faustman DL, Wang L, Okubo Y, Burger D, Ban L, et al. (2012) Proof-ofconcept, randomized, controlled clinical trial of Bacillus-Calmette-Guerin for treatment of long-term type 1 diabetes. PLoS One 7: e41756.

7. Ban L, Zhang J, Wang L, Kuhtreiber W, Burger D, et al. (2008) Selective death of autoreactive T cells in human diabetes by TNF or TNF receptor 2 agonism. Proc Natl Acad Sci U S A 105: 13644-13649.

8. Hamano R, Huang J, Yoshimura T, Oppenheim JJ, Chen X (2011) TNF optimally activatives regulatory $T$ cells by inducing TNF receptor superfamily members TNFR2, 4-1BB and OX40. Eur J Immunol 41: 2010-2020.

9. Ryu S, Kodama S, Ryu K, Schoenfeld DA, Faustman DL (2001) Reversal of established autoimmune diabetes by restoration of endogenous beta cell function. J Clin Invest 108: 63-72.

10. Tracey KJ, Beutler B, Lowry SF, Merryweather J, Wolpe S, et al. (1986) Shock and tissue injury induced by recombinant human cachectin. Science 234: 470474

11. Wong GH, Goeddel DV (1986) Tumour necrosis factors alpha and beta inhibit virus replication and synergize with interferons. Nature 323: 819-822.

12. Rahman MM, McFadden G (2006) Modulation of tumor necrosis factor by microbial pathogens. PLoS Pathog 2: e4.

13. Clark IA (2007) How TNF was recognized as a key mechanism of disease Cytokine Growth Factor Rev 18: 335-343.

14. Kühtreiber WM, Hayashi T, Dale EA, Faustman DL (2003) Central role of defective apoptosis in autoimmunity. J Mol Endocrinol 31: 373-399.

15. Burger DE, Wang L, Ban L, Okubo Y, Kühtreiber WM, et al. (2011) Nove automated blood separations validate whole cell biomarkers. PLoS One 6: e22430.

16. De Paschale M, Clerici $P$ (2012) Serological diagnosis of Epstein-Barr virus infection: Problems and solutions. World J Virol 1: 31-43.

17. Diaz D, Prieto A, Barcenilla H, Monserrat J, Prieto P, et al. (2004) Loss of lineage antigens is a common feature of apoptotic lymphocytes. J Leukoc Biol 76: 609-615

18. Strachan DP (1989) Hay fever, hygiene, and household size. BMJ 299: 1259 1260.

19. Knip M (2012) Descriptive epidemiology of type 1 diabetes--is it still in? Diabetologia 55: 1227-1230.

20. Marshall AL, Chetwynd A, Morris JA, Placzek M, Smith C, et al. (2004) Type 1 diabetes mellitus in childhood: a matched case control study in Lancashire and Cumbria, UK. Diabet Med 21: 1035-1040.

21. Cardwell CR, Carson DJ, Yarnell J, Shields MD, Patterson CC (2008) Atopy, home environment and the risk of childhood-onset type 1 diabetes: population-based case-control study. Pediatr Diabetes 9: 191-196.

22. (2000) Variation and trends in incidence of childhood diabetes in Europe EURODIAB ACE Study Group. Lancet 355: 873-876.

23. Airaghi L, Tedeschi A (2006) Negative association between occurrence of type 1 diabetes and tuberculosis incidence at population level. Acta Diabetol 43 $43-45$.

24. Keijzer C, van der Zee R, van Eden W, Broere F (2013) Treg inducing adjuvants for therapeutic vaccination against chronic inflammatory diseases. Front Immunol 4: 245.

25. van Eden $W$, van Herwijnen $M$, Wagenaar $J$, van Kooten $P$, Broere $F$, et al. (2013) Stress proteins are used by the immune system for cognate interactions with anti-inflammatory regulatory T cells. FEBS Lett 587: 1951-1958.

26. Chen X, Wu X, Zhou Q, Howard OM, Netea MG, et al. (2013) TNFR2 is critical for the stabilization of the CD4+Foxp3+ regulatory $T$. cell phenotype in the inflammatory environment. J Immunol 190: 1076-1084.

27. Faustman DL (2013) EBV infection and anti-CD3 treatment for Type 1 diabetes: bad cop, good cop? Expert Rev Clin Immunol 9: 95-97. 